and, although the bullet was large, the wound would not easily admit the little finger.

On consultation with Staff-Surgeon Bell-Murray, R.N., who came soon after my arrival, we probed the wound slightly in order to have an idea of the direction of the bullet, and we ascertained that its course was almost directly inwards, and a little downwards and forwards. From the action of the bladder, which we looked upon as reflex, it was surmised that some part of the urinary apparatus was involved, especially as the wound was near the kidney, and was directed towards the distended bladder

A few hours after the injury, he had rallied so much from the shock as to admit of his being removed to bed. The round was dressed with simple carbolised dressing, a full hypodermic injection of morphia having been previously administered. I may here mention that he was kept fully under the influence of the morphia for ten days after the injury, as it was feared the intestines were injured.

On visiting him again in the evening, I found him more comfortable. Temperature $100^{\circ}$; pulse 105, weak, and slightly "thready"; mind quite clear, and complained of little pain. He had passed no urine in the meantime. On the following morning, there were decided symptoms of peritonitis; his urine was distinctly bloody, but was passed with little pain. His abdomen was distended and painful, and the pain was most marked between the umbilicus and symphysis pubis. Temperature $100.2^{\circ}$; pulse I05. During the succeeding three days, the symptoms did not vary much, but the tympanites was exceedingly troublesome. The pulse varied from IOO to IIO, and the temperature from $100^{\circ}$ to $101.5^{\circ}$.

The urine always contained blood. Turpentine stupes afforded relief to the tympanites, and tincture of the perchloride of iron ( 15 minims three times a day) and gallic acid were prescribed for the hromaturia. His diet was composed of slops, excluding all preparations of animal food. He was allowed to drink large quantities of mineral waters mixed with milk.

The whole aim of treatment during this time, and for the first week, was to restrain peristaltic action by the hypodermic injection of morphia two or three times a day.

Beyond a little flatus, there were no indications of any action of the bowels until the fifteenth day, when he received an injection of thin gruel and an ounce of castor-oil. This injection was retained for thirtysix hours, until brought away by a further one of a pint and a half of tepid water, and with it came several hardened scybala, which caused a good deal of pain and disturbance.

On the eighteenth day, the morning temperature was $\mathrm{IO}^{\circ} . \mathrm{He}$ received an occasional erema for the succeeding few days, with little effect until the twentieth day, when I gave him internally an ounce of castor-oil, which was soon followed by a copious evacuation, almost normal in appearance. His temperature immediately fell to $99.8^{\circ}$, from $10 I^{\circ}$ on the preceding day.

From the twenty-first to twenty-fifth day, the urine was gradually becoming clearer ; but, on the afternoon of the latter day, I received an urgent summons to attend him, and on my arrival I found him extremely collapsed. He had just passed, by the urethra, about thirty ounces of arterial blood without any admixture of urine, doubtless from ulceration of one of the vessels of the kidney. His attendant told me that it came in a free stream, but immediately coagulated in the utensil, and that the patient fainted while the expulsive efforts of the bladder were being continued. His pulse was scarcely appreciable; temperature $98 . \mathrm{I}^{\circ}$; face perfectly colourless.

For the succeeding four days, his condition was extremely critical, from the large quantities of blood he had been passing daily, or rather hourly, for his bladder was very irritable. A large catheter was occasionally passed to wash out the bladder, and this process was unattended by pain. The gallic acid was increased to ro grains every three hours, and the tincture of iron given as before.

From the thirtieth day, a gradual improvement was registered; and, on the thirty-fifth day, his urine was again normal, as were also his pulse and temperature.

Beyond a few accidental changes of temperature, his progress towards recovery was uninterrupted from this day. On the fiftieth day, he was allowed to leave his bed for an easy-chair; and, sixty-two days after the injury, he departed for his home, a distance of more than two hundred miles. The wound had perfectly healed up. Nothing remained but 2 hardened cicatrix, which was not tender to the touch.

Since this report was written, I have heard from the patient. His letter was dated more than six months after the injury. He is "regaining strength daily, but is not yet able for manual labour".

REMARKS. - There are some interesting points to be considered in connection with the foregoing case.

I. The man's future. What value, for instance, would an insurance office set on his life? He has a bullet in his abdomen, probably in or near the left kidney. The latter organ was certainly injured, for a wound of any other part of the urinary tract would not account for the large quantity of blood passed by the urethra on the twenty-fifth day. Is there any danger of the bullet being displaced by gravity, or muscular action, or some other cause, and giving further trouble?

2. The peritonitis resulting from the wound was comparatively slight, and did not give any trouble after the fourth day. The descending colon seemed to have escaped injury altogether.

3. I regard the vomiting after the injury as exceedingly fortunate, as he had just partaken of a hearty dinner of boiled beef and vegetables.

4. The $B . P$. hypodermic injection of morphia acted very satisfactorily, and effectually paralysed the action of the bowel for the first fortnight. As a rule, each hypodermic injection was succeeded by a comfortable sleep of some hours' duration.

\section{RUPTURE OF THE HEART.}

\section{By NEIL MACLEOD, M.D., Shanghai.}

THE following case, from the absence of symptoms during life, the well marked post mortem lesions, and the mode of termination, is, I think, worthy of record.

G. W. B., a short, stoutly built man aged 58 , was seized, on July 4 th, at 5 A.M., with a violent pain in the chest. IVhen I saw him at 8.30 A.M., he had a very anxious look, and complained of intense pain, confined to the middle line, along the whole length of the sternum; also of a little difficulty of breathing, and weakness of voice. There was no lividity. The right pulse could scarcely be felt ; the left was weak, but quite distinct. There was no impulse in the episternal notch, nor was there any abnormal dulness. The heart-sounds were feeble, and no bruit could be heard. The breathing was laboured and wheezy, and made the examination of the heart somewhat difficult. A quarter of a grain of sulphate of morphia was given hypodermically.

At noon he expressed himself as feeling better, his breathing as easier, and the pain as much less. The left pulse was fuller than in the morning, the right remaining as before. Ile did not know whether there was usually any difference in the wrist pulses; and added that he had been quite well up to the time of seizure. His heart had never been examined, and he had never felt anything unusual in that region.

He died suddenly at 6 P.M. A friend who had been sitting by him at the time stated that, after expressing himself as feeling much better he laid his head down as if to sleep, became black in the face, and was dead-all in the interval between the first and the fourth guns of a salute which was then being fired, that is, in about two minutes. An hour after death, the face, and particularly the lips, were unusually pale.

Post mortem examination on the following morning at 7. Rigor mortis was well marked. The lungs, liver, spleen, and kidneys were healthy. Puncture of the pericardium was followed by the escape of bloody serum; and the heart lay embedded in a firm dark clot which, with the serum, filled a large breakfast cup. On the middle of the anterior wall of the left ventricle, a dark line, about one inch long, ran almost transversely; no distinct opening could be made out in it. The heart was then removed, the aorta slit down to its valves, and water, poured into the heart from above, slowly oozed through tiny openings in the dark line. The heart was well covered with fat except over the rent, and seemed of natural size. There were a few atheromatous plates in the aorta and at the attachments of the aortic valves. Hard nodules could be felt in the thickened mitral valves. The wall at the rent measured about one-and-a-half lines; at the apex half an inch thick. In the tissue surrounding the rent, extravasated blood was seen on section to extend for half an inch in every direction, and dark blood clot was firmly plastered over a corresponding area on the internal surface. On section of the apex, at some points, it was impossible to distinguish the inner limit of the fat covering, white patches shading off into the muscular tissue, firmer, however, than the fat, and offering resistance to the knife. There was no naked eye sign of fatty degeneration. The left coronary artery and its chief branch could be traced by the fingers among the fat, being atheromatous and in some places nodulated. On dissecting these vessels out, and slitting them up, at the nodules were small half decolorised clots filling up a much diminished lumen. The rent lay in the angle between the artery and its branch.

At a microscopic examination in the forenoon of the same day, specimens taken from a healthy-looking papillary muscle presented some of the muscular fibre perfectly striated; others had the striæ partially obscured by minute fatty granules; while the fibres themselves were perfectly 
distinct from one another. Specimens from the neighbourhood of the rent presented fibres with the striation in some absent, in others indistinct, all containing more or less fatty granules, the fibres themselves being much less distinct than in the more healthy specimens. Specimens from the whitish tissue of the apex, teazed with difficulty, presented no trace of muscular fibre, and consisted of fine white fibrous tissue with here and there fatty granules.

REMARKS. - This case then is a well marked example of five different pathological cardiac conditions, without any history of heart trouble till the day of death : $\mathbf{I}$. Atheroma of aorta, aortic valves, and coronary arteries; 2. Chronic myocarditis; 3. Chronic endocarditis ; 4. Fatty degeneration, probably the result of the diseased coronary arteiies; 5. Rupture of the left ventricle.

Living an easy life, in charge of an opium receiving ship, having no stairs to climb, no hill or even incline within thirty miles of Shanghai, it is probable that the patient's heart had never been severely tried. I may add that he told me he had had "a good stool the first thing in the morning as usual "; but whether this was before or after the onset of the pain, I neglected to inquire.

The microscopic specimens were mounted in Farrant's solution, and, on examination a week later, had almost ceased to be distinctive of fatty degeneration, having been perfect demonstrations when freshly put up. I understand that there is no known method of preserving the microscopic appearance of this change.

\section{NOTES ON INTERMITTENT BREATHING.}

\section{By S. WILSON HOPE, L.R.C.P., Petworth.}

IN the following notes, intermittent breathing is considered $(a)$ as coexistent with senile bronchitis, $(b)$ with hysteria, $(c)$ with inhalation of anæsthetics, (ci) with cardiac dropsy, and in view of its clinical importance-hardly second to that of intermittent heart. Some inquiry is made into its causation.

$a$. There is a lean old lady, upwards of eighty years old, whose pulse has rarely been regular any time when $I$ have felt it during the past seven years, who caught a cold; and a bad cough, with spitting of a good deal of frothy, and later of sticky, mucus, came on, and great weakness. Before the attack, she used to spend her nights sitting more or less propped up in bed, till sleep overcame her, when she usually fell over to one side, lying sleeping with the head quite low. During the early part of her illness, her nights were very restless, as were the days too; but later, for many hours during the day, through many days and weeks, she lay, taking but little notice of anything or anybody, though showing at intervals by a remark that her mind was quite clear for the moment. Very early in her illness, her niece, who nursed her, had to remove the phlegm from her mouth when she coughed; medicine had to be given up, and threatening bed-sores to be treated by a water-bed. Soon the breathing became labouring and still by turns, rhythmically; the pauses, or quasi-pauses, growing so long, that once or twice her nurse thought she had ceased to live and then food of all sorts began to be avoided, and death calmly awaited, indeed desired by her. So day after day passed, till at last a turn for the better took place; and now, though she is too weak to walk alone, her mind is as active as ever, and her breathing and pulse as before her illness.

In the same way, an old man of eighty-four or five had an illness marked in part by this peculiar intermittent breathing, which ended in recovery of his usual health; though he, too, had a very irregular and intermittent heart.

b. Many women and some men are occasionally seized in some way that they fall usually backwards, when, if watched, it will be seen that for a time the breathing is stopped, and there is quiet of muscles generally; then a period of struggling follows; then a deep drawn sigh, or several, along with incoherent talking, crying, etc.; and then either the person goes on breathing better till respiration is natural, and health is regained; or, more commonly, another pause occurs in the breathing, and a fresh attack often runs through a similar course. If we almost anticipate the pause in the breathing, and excite an inspiration, as by pouring or dashing cold water on the face just at the right time, we may often cut short an attack of this kind.

c. Again, it is curious to mark how closely this kind of fit mocks the early effects of inhaling chloroform or ether: there are the pauses, sometimes even fatal; the strugglings, and the incoherent talking, crying, etc. But the best of this class of cases is, that it seems to suggest a good working hypothesis for the whole, viz., that direct irritation of the terminal fibres of the superior laryngeal nerve is the cause of the temporary or permanent stoppage of the breathing during the inhalation of anæsthetics. But of this presently. d. We often find cases of cardiac dropsy marked by this rhythmical succession of breath-pause and breath-struggle. On watching, it is seen that the first breath drawn is followed by a rising series, in which each breathing is deeper than the foregoing till a deepest is reached; then come a falling series of shallower and shallower breathings, till the respiratory movements cease for a while. These cases, too, end sometimes in the dropsy disappearing, and the patient's getting about again.

Now, with respect to the causation of intermittent breathing, it seems to me obscure, and in want of experiments; but, adopting the method of exclusion, we find, first, that it is not caused by the cardiac disturbance, though often associated with it, because an intermittent and irregular heart is often seen in trifling cases of indigestion, where the breathing is natural; and conversely, the pulse may be steady and regular, as in some cases of apoplexy, which become marked by pauses in the breathing, though never, so far as I know, of the same peculiar rhythm. Nevertheless, they go to show that no causal connection exists between the heart and breathing troubles.

Secondly, the pauses are clearly not instances of true apnœa, that is, in which for a time the blood is saturated with oxygen; as we have only to look at the lips of our patients to see that such an idea is absurd.

Thirdly, it døes not seem to run with ordinary notions that this peculiar respiration, made up of pauses and hard breathings following each other in regular order, could be a sign of some organic change in the respiratory centre; inasmuch as such fundamental things as respiratory cardiac and vaso-motor centres, though thought liable to be stopped somehow at death, seem in themselves to be treated as above disease, just as they transcend in their effects common notions of matter and organisation.

Fourthly, it is not easy to hit upon a scheme of reflex nerve-action which will account for this breathing. Hering's experiments, showing that distending the lung with air excites fibres in the vagi, which so act on the respiratory centre as to stop respiration, hardly helps us to explain the pauses, much less their rhythm; because, though truly the hard breathings fill the lungs with air, they do not overfill them as in Hering's experiments.

Fifthly, the canon "that the activity of the respiratory movements varies inversely as their oxidating effect on the blood" does not enable us to see our way through the rhythmic hard breathings.

Enough has been said to show that the subject is obscure, and I shall be glad if this paper should lead someone to let in a little more light upon it.

\section{A CASE OF ADDISON'S DISEASE, ASSOCIATED WITH TUBERCULOSIS OF THE LUNGS AND KIDNEY.}

By HERBERT E. WRIGHT, M.R.C.S., L.R.C.P., etc.

Henry C. B., aged 8, came under my care on April 17 th, I880. His father and mother were quite healthy; there were also six brothers and sisters in good health. His mother's family were remarkably phthisical -her mother, sisters, brothers, and one or two uncles having died of phthisis.

The patient had enjoyed good health up to three years ago, when he began to suffer from cough, night sweats, and loss of flesh, for which he was treated in Liverpool for about nine months, with little benefit. About two years ago, he began to suffer from pain in his right loin; shortly after a swelling appeared at this spot. This gradyally increased in size, and became painful; on account of which the patient was taken to another medical man, who ordered the swelling to be poulticed, which was continued for three months, when the swelling burst, and discharged a considerable quantity of pus through four openings. Three of these sinuses healed up after about two months, but the fourth never healed completely, but still continued to discharge pus. The patient now began to improve considerably ; his appetite returned; he regained flesh ; and, with the exception of a slight cough and expectoration, he appeared to be quite well. His mother told me that the boy continued to improve in health until six weeks before he came under my charge, when he accidentally received a kick in his right loin, which reopened the sinuses, and caused them to discharge pus freely again. His urine at this time was thick, and offensive. His cough now became more troublesome, and he rapidly began to lose flesh. He never spat blood, but he had occasional attacks of diarrhœea.

When I saw the patient on April I7th, I880, he was extremely ema. ciated, and very tubercular-looking. I noticed about a dozen small pigment-spots scattered over the face, which, the mother informed me, had made their appearance during the last three months. The skin of the face was not much darkened; but the neck, the backs of both hands 